\title{
Resource allocation and post-reproductive degeneration in the freshwater cnidarian Hydra oligactis (Pallas, 1766)
}

\author{
Jácint Tökölyi *, Zsófia Ôsz, Flóra Sebestyén, Zoltán Barta \\ MTA-DE “Lendület” Behavioral Ecology Research Group, Department of Evolutionary Zoology, University of Debrecen, Egyetem tér 1, 4032 Debrecen, Hungary
}

\section{A R T I C L E I N F O}

\section{Article history:}

Received 2 April 2016

Received in revised form 22 May 2016

Accepted 22 June 2016

Available online $\mathrm{xxx}$

\section{Keywords:}

Cnidaria

Food availability

Life history

Senescence

Survival/reproduction trade-offs

\begin{abstract}
A B S T R A C T
Freshwater hydra are among the few animal groups that show negligible senescence and can maintain high survival and reproduction rates when kept under stable conditions in the laboratory. Yet, one species of Hydra (H. oligactis) undergoes a senescence-like process in which polyps degenerate and die after sexual reproduction. The ultimate factors responsible for this phenomenon are unclear. High mortality in reproducing animals could be the consequence of increased allocation of resources to reproduction at the expense of somatic maintenance. This hypothesis predicts that patterns of reproduction and survival are influenced by resource availability. To test this prediction we investigated survival and reproduction at different levels of food availability in 10 lineages of $\mathrm{H}$. oligactis derived from a single Hungarian population. Sexual reproduction was accompanied by reduced survival, but a substantial proportion of animals regenerated after sexual reproduction and continued reproducing asexually. Polyps belonging to different lineages showed differences in their propensity to initiate sexual reproduction, gonad number and survival rate. Food availability significantly affected fecundity (number of eggs or testes produced), with the largest number of gonads being produced by animals kept on a high food regime. On the other hand, survival rate was not affected by the amount of food. These results show that survival is conserved at the expense of reproduction in this population when food is low. It remains a question still to be answered why survival is prioritized over reproduction in this population.
\end{abstract}

(C) 2016 Published by Elsevier Ltd.

\section{Introduction}

Cnidarians display one of the simplest but most versatile body organizations within the animal kingdom, characterized by high tissue plasticity and extraordinary regenerative capabilities (Holstein et al., 2003). Perhaps due to these regenerative capabilities, some members of this phylum display very low rates of senescence (Brock and Strehler, 1963; Martıńez, 1998; Boehm et al., 2013; Schaible et al., 2014, 2015). In a recent study, for instance, Schaible et al. (2015) have shown that Hydra magnipapillata and Hydra vulgaris maintained constant rates of fertility and mortality in the laboratory over a period of eight years.

Despite their ability to forego senescence, some Hydra species under specific conditions show increasing rates of age-dependent mortality along with a senescence-like degeneration. As first observed by Brien (1953) and later studied by Yoshida et al. (2006), individuals of $H$. oligactis initiate sexual reproduction when the temperature is reduced, after which they die within a few months. Sexual reproduction is followed by a reduction in the number of interstitial stem cells, a decline in the rate of food capture and contractile movements, a decrease in body size and an exponential increase in mortality rate (Yoshida et al., 2006). The reasons why H. oligactis undergoes this senescence-like degeneration are unclear.

\footnotetext{
* Corresponding author

Email address: jtokolyi@vocs.unideb.hu (J. Tökölyi)
}

According to the disposable soma theory of aging, resources invested into the maintenance of the soma are traded off against investment into reproductive functions (Kirkwood and Rose, 1991). As a consequence, animals with high reproductive investment are expected to show lower levels of self-maintenance and higher levels of aging, a prediction that has received broad support (summarized in Boggs, 2009). Although few systematic studies have been performed on reproductive investment in Hydra species, studies generally report a higher number of reproductive organs (testes and ovaries) in $\mathrm{H}$. oligactis compared to other species (e.g., Schuchert, 2010). This raises the possibility that the post-reproductive degeneration observed in this species might be caused by an increased allocation of resources to reproduction at the expense of self-maintenance functions. Indeed, Reisa (1973) suggested that the "depression" observed in sexually reproducing $H$. oligactis might be the consequence of interstitial stem cells being converted into germ cells instead of nematocysts, which would prevent feeding throughout the sexual cycle.

If post-reproductive degeneration in hydra is caused by the trade-off between survival and reproduction, then the amount of resources available to the animals is expected to influence investment into these functions, with several possible outcomes. First, animals facing a resource shortage might reduce their reproductive investment to increase survival. In some cases experiencing periods of low food availability results in higher levels of stress tolerance and lower rates of aging, a phenomenon termed dietary restriction (e.g., Masoro and Austad, 1996; Partridge et al., 2005; Walker et al., 2005). However, such an effect does not occur in all species (Nakagawa et al., 2012). 
Within rotifers, for instance, closely related species may show increased or decreased longevity when exposed to the same food restriction treatment (Kirk, 2001). In hydra there is no evidence so far for dietary restriction-mediated increases in self maintenance levels (Bridge et al., 2010; Tökölyi et al., 2016), although species differ in the way in which oxidative stress tolerance is maintained in the face of reduced food availability (Tökölyi et al., 2016). Secondly, food shortage might signal increased future mortality risk, resulting in higher investment into reproduction and a reduction in survival, a strategy termed "terminal investment" (Clutton-Brock, 1984; Fischer et al., 2009; McNamara et al., 2009). Such a strategy is seen, e.g., in some rotifers (Kirk, 2001; Stelzer, 2001) and birds (Velando et al., 2006), which increase reproductive effort when food availability becomes limited or their immune system is experimentally challenged. Thirdly, in the most simplistic scenario, food shortage may reduce both reproduction and survival at the same time.

To test which of these scenarios, if any, occurs in H. oligactis undergoing post-reproductive degeneration, we individually followed hydra polyps kept at different levels of food supply and measured fecundity and survival as two opposing facets of life history trade-offs. Fecundity was quantified as number of eggs in females, while in males we used the number of testes as a proxy for gamete production. We predicted that fecundity and post-reproductive lifespan would be concomitantly reduced if both functions are valued in the same way, while departure from a parallel reduction would indicate that one of the functions is preserved at the expense of the other, giving rise in the most extreme cases to "dietary restriction" (survival increased and fecundity reduced) or "terminal investment" (fecundity increased and survival reduced) effects.

\section{Materials and methods}

\subsection{Animals and culture conditions}

Animals for the present study were derived from ten polyps collected in May 2015 from an oxbow lake of the Tisza river near Tiszadorogma, Hungary (47.67N, 20.86E; Fig. 1.). We collected animals that were at least $2 \mathrm{~m}$ apart to increase the chance that both males and females were found and to reduce the chance that multiple asexual members of the same genetic clone were collected. Species identity was determined based on morphological traits and investigation of nematocysts (under $400 \times$ magnification in a light microscope) based on Schuchert (2010). H. oligactis can be distinguished morphologically from other hydra species by the presence of a distinct stalk in the foot region, very long tentacles and asymmetric emergence of tentacles in the buds. We note, however, that species delimitation in Hydra is far from a resolved issue (see, e.g., the recent study of Schwentner and Bosch, 2015), hence the possibility that this population belongs to a distinct branch of the 'oligactis' group cannot, at present, be excluded.

After collection, animals were moved to the laboratory and kept under standardized conditions $\left(20{ }^{\circ} \mathrm{C}\right.$ temperature, $12 / 12 \mathrm{~h}$ photoperiod, in a standard hydra medium containing $1.0 \mathrm{mM} \mathrm{CaCl}_{2}, 0.1 \mathrm{mM}$ $\mathrm{MgCl}_{2} \cdot 6 \mathrm{H}_{2} \mathrm{O}, 0.03 \mathrm{mM} \mathrm{KNO}_{3}, 0.5 \mathrm{mM} \mathrm{NaHCO} \mathrm{Na}_{3}$ and $0.08 \mathrm{mM}$ $\left.\mathrm{MgSO}_{4}\right)$. We propagated these animals asexually by feeding them three times a week with freshly hatched Artemia nauplii. This propagation phase lasted for approximately two months, during which the animals were kept in 6-well culture plates in groups of three hydra specimens per well (all three belonging to the same clone).

At the start of the experiments polyps were moved to 24-well plates (1 polyp per well, individuals within a plate belonging to one or two clones). 36 polyps from each original clone were used, resulting in a starting cohort of 360 individuals. To facilitate cleaning, we used special plates with holes on the bottom of the wells forming a sieve (each well had 17 holes with a diameter of $0.7 \mathrm{~mm}$ on the bottom). The holes were too small for hydra to pass, but large enough to let uningested Artemia nauplii pass through when the plate was raised from the water. The plates were kept individually in plastic containers with a flat bottom and immersed in hydra medium (wells were approximately half full and polyps were kept in $\sim 1.5 \mathrm{ml}$ medium). A piece of glass (exactly fitting the bottom of the plates) was put below the plates to block experimental animals, detached buds or eggs leaving the wells when the plates were not moved. For cleaning, the plates (with the hydra) were simply taken out of their containers and moved to clean containers with fresh medium. To prevent the accumulation of bacteria, we moved hydra polyps to clean plates once every two weeks.
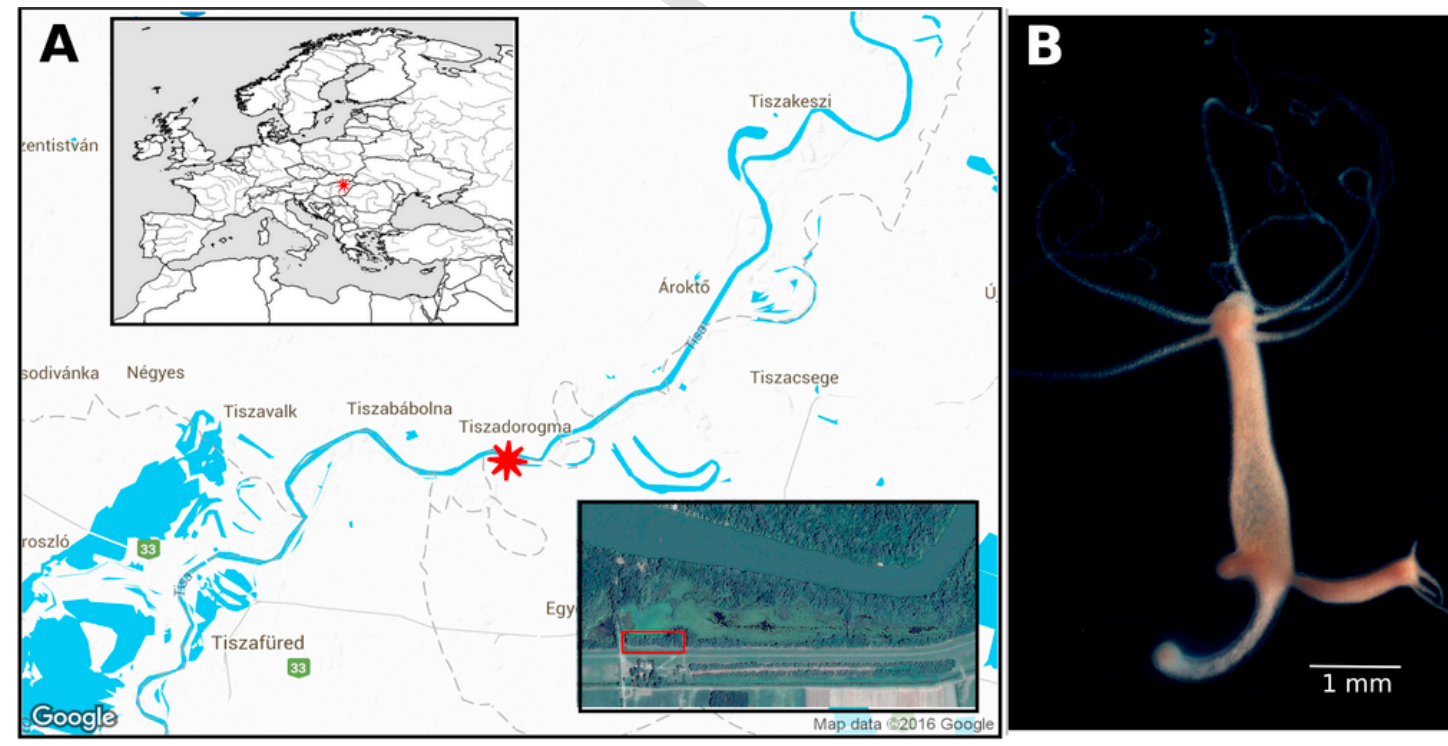

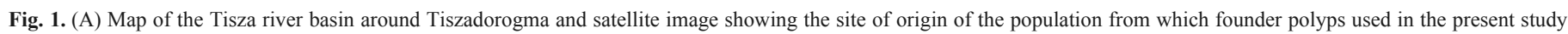
were collected (marked by a red star on the map and a red rectangle on the inset). (B) Asexual H. oligactis polyp from the Tiszadorogma population. 
We fed the animals three times a week from a dense Artemia slurry using a micropipette, as described previously (Tökölyi et al., 2016). Depending on their treatment, they received either 3, 6, 10 or $20 \mu \mathrm{l}$ Artemia suspension on each occasion $(1 \mu \mathrm{l}$ contained approximately 7-8 nauplii). Food treatments were distributed in plates according to a block design (all four treatments present in every plate). Newly detached buds were counted and removed prior to feeding. After feeding, plates with hydra were moved to containers with fresh medium. Experimental animals were kept under these conditions for two weeks. After that, we lowered the temperature to $7{ }^{\circ} \mathrm{C}$ (temperatures below $12{ }^{\circ} \mathrm{C}$ are used to induce sexual reproduction in $\mathrm{H}$. oligactis; Littlefield et al., 1991; Yoshida et al., 2006; Kaliszewicz and Lipińska, 2012; Kaliszewicz, 2015). We also switched photoperiod to a $8 / 16 \mathrm{~h} \mathrm{light/dark}$ cycle because there are observations suggesting that a short photoperiod might induce sexual reproduction as well (Reisa, 1973).

To quantify the intensity of sexual reproduction we recorded the number of detached eggs in females and the number of testes in males. Females continually produce and shed eggs during their sexual phase, while males develop several distinct testes along the body column that continuously produce sperm (Reisa, 1973). We counted the number of detached eggs in females three times per week, prior to feeding. Care was taken not to disturb the plates so that detached eggs did not escape through the holes at the bottom of the wells. The number of testes in male polyps was counted once a week under a stereo microscope.

Death was recorded when the animals disintegrated or disappeared between feedings. We were extremely careful to retain experimental animals even if they shrank to a very small size, always checking the plates and the containers in case of a potential death event. Four animals were accidentally lost and we excluded them from all subsequent analyses.

\subsection{Statistical analyses}

We analyzed data using generalized linear models (GLMs) or generalized linear mixed models (GLMMs) implemented in the R statisti- cal environment ( $\mathrm{R}$ Core Team, 2014), using the lme4 R package for GLMMs (Bates et al., 2015). We started by testing whether individual clones differed in their propensity to start sexual reproduction, number of gonads and survival in GLMs with these variables as dependent variables and clone ID as fixed predictor. GLMs with a Poisson distribution were used to analyze fecundity (maximum number of testes per male polyp, total number of eggs produced by a female polyp during the sexual period), while the occurrence of sexual reproduction and survival was analyzed with binomial GLMs (both variables have a binomial outcome: sexual reproduction observed or not, and polyps survived or died, respectively). Next, we tested the effect of food treatment on the dependent variables in GLMMs (with either Poisson or binomial distribution). GLMMs were required in this case to take into account that our experimental animals did not represent independent data points (polyps belonging to a given clone are more similar to each other than expected by chance). Hence, in these analyses clone ID was included as a random variable, in addition to treatment as a fixed variable. Lastly, we used GLMMs to test the relationship between fecundity and survival rate. Significance of fixed variables was tested via likelihood ratio tests (LRTs), by comparing models containing the fixed effect to simplified models without it.

\section{Results}

The pattern of asexual and sexual reproduction and survival changed over the course of the experiment as shown in Fig. 2. Lowering the temperature inhibited budding for approximately two weeks; then animals produced buds again for two weeks, which was followed by initiation of sexual reproduction. Of the ten clones five were found to be males and five to be females. The number of days elapsed from the start of the temperature manipulation to the initiation of sexual reproduction was $38 \pm 3.91$ days in males and $47 \pm 5.65$ days in females (mean \pm SD in both cases). Survival rate started to decrease after the initiation of sexual reproduction, but a substantial proportion of animals survived and started budding again. We stopped the experiment after 150 days, when most of the animals were either dead

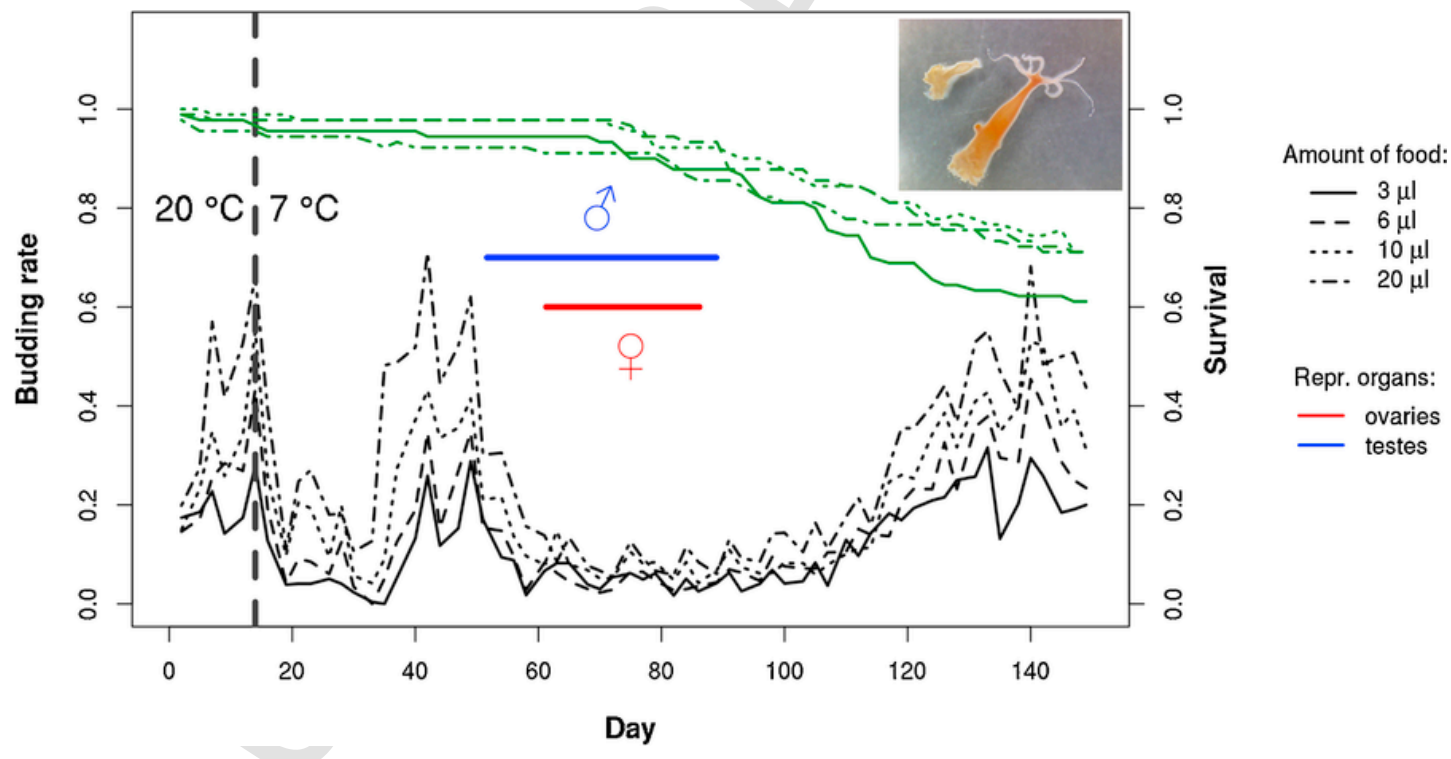

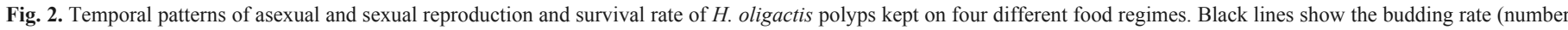

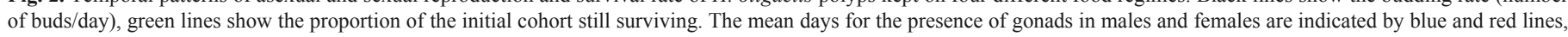
respectively. The inset shows two polyps towards the end of the experiment in different stages of degeneration; the individual on the right regenerated almost completely. 
( $N=109,31 \%$ of the initial cohort) or had regenerated after sexual reproduction and produced at least one bud $(N=176 ; 49 \%)$. The remainder either did not initiate sexual reproduction until the end of the study $(N=33,9 \%)$ or were in an intermediate state (i.e., survived until day 150 but did not produce any buds following sexual reproduction; $N=38,11 \%$ ). We scored these intermediate animals as survivors (even though some of them might have died later without regenerating) because they fulfilled the criterion of surviving until day 150 . We found no bias in the distribution of these animals according to food treatment $(N=8,6,12,12$ animals out of $N=55,64,64,64$ surviving animals in the 3, 6, 10, $20 \mu \mathrm{l}$ Artemia groups, respectively; Fisher's exact test, $p=0.539$ ).

Individual clones differed significantly in their propensity to initiate sexual reproduction (binomial GLM, deviance $=163.62, \mathrm{df}=9$, $p<0.001$; Fig. 3A) and in the probability of surviving to the end of the experiment (binomial GLM, deviance $=75.325, \mathrm{df}=9, p<0.001$; Fig. 3B). The number of testes produced by males was marginally sig-
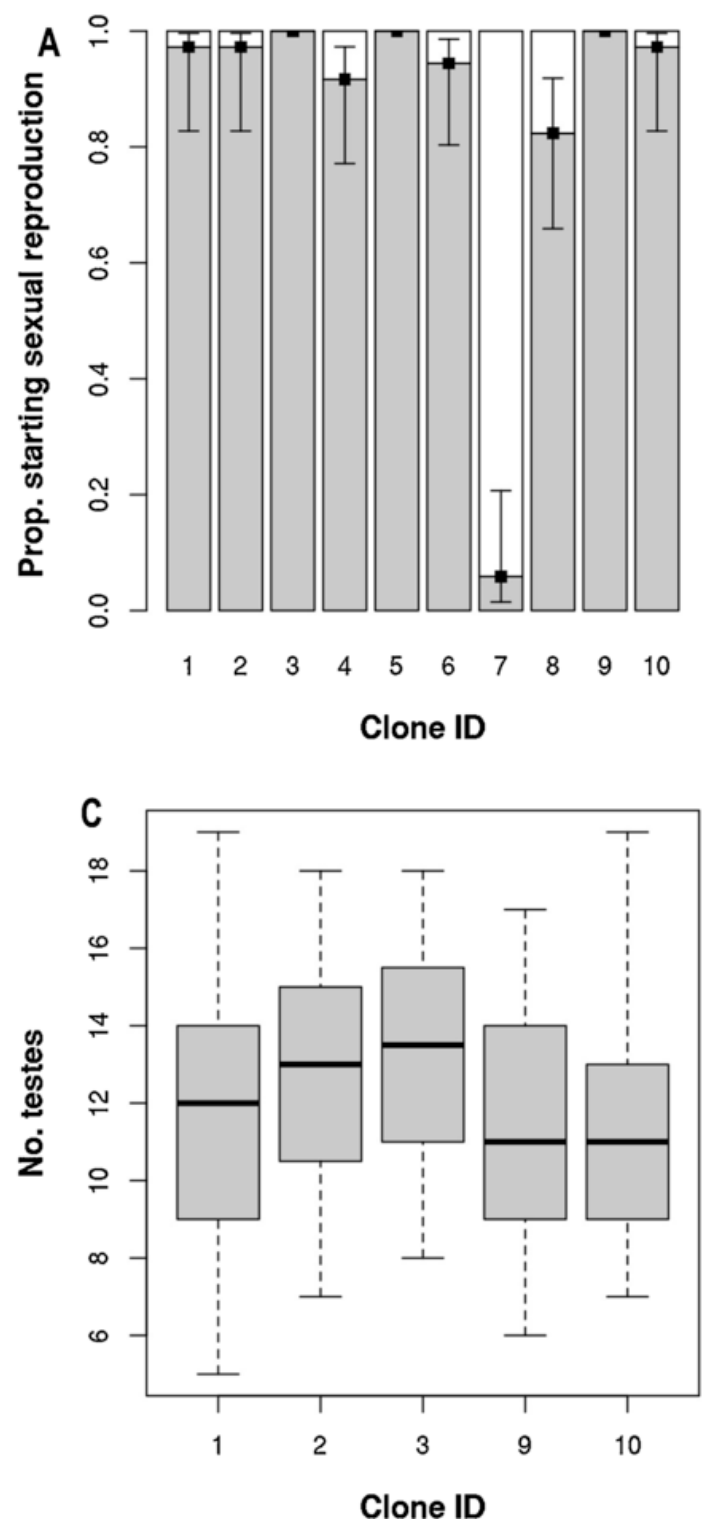

nificantly different between clones (Poisson GLM, deviance $=9.112$, $\mathrm{df}=4, p=0.058$; Fig. $3 \mathrm{C}$ ). The total number of detached eggs significantly differed between female clones (Poisson GLM, deviance $=35.952, \mathrm{df}=4, p<0.001$; Fig. $3 \mathrm{D})$.

Food treatment had no effect on the occurrence of sexual reproduction (binomial GLMM, $\chi^{2}=2.829, \mathrm{df}=3, p=0.419$ ), but it did have a significant effect on the number of testes in males (Poisson GLMM, $\chi^{2}=69.454, \mathrm{df}=3, p<0.001$; Fig. $3 \mathrm{~A}$ ) and the number of eggs in females (Poisson GLMM, $\chi^{2}=54.015$, $\mathrm{df}=3, p<0.001$; Fig. 4B). Survival probability was not affected by food treatment (binomial GLMM, $\chi^{2}=3.854, p=0.278$; Fig. $3 C$ ). Furthermore, survival probability was not related to fecundity neither in males (maximum number of testes, binomial GLMM, $\chi^{2}=1.800, p=0.180$ ) nor in females (total number of eggs, binomial GLMM, $\chi^{2}=0.537, p=0.464$ ). Lastly, excluding polyps that survived until the end of the experiment but did not regenerate following sexual reproduction ( $N=38$ animals) had no qualitative effect on our results (not shown).
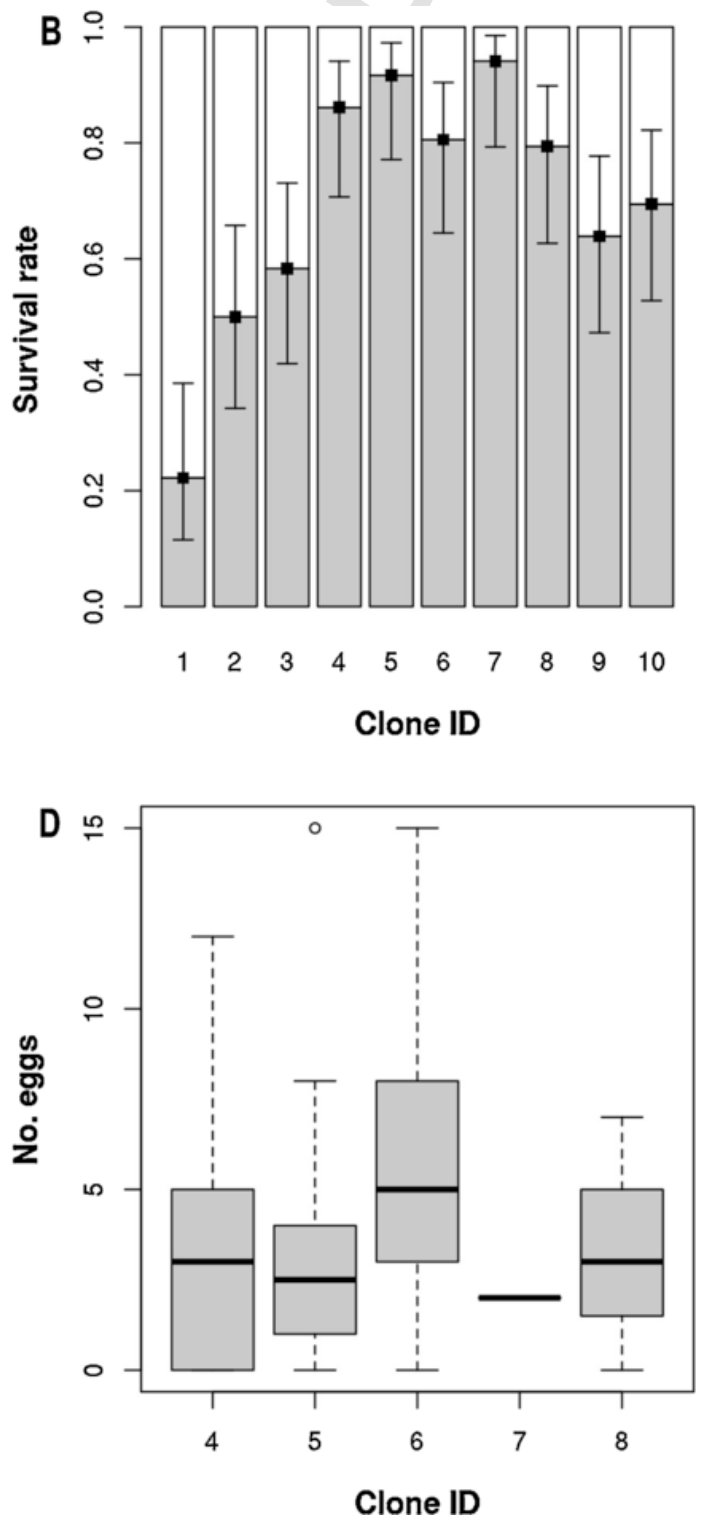

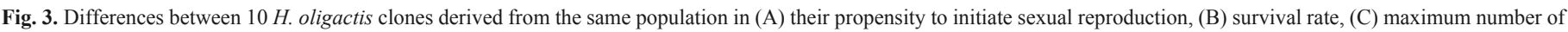

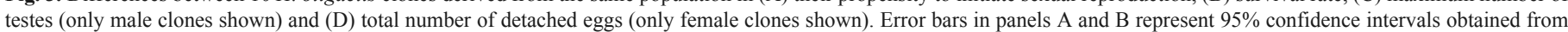
binomial GLMs. 


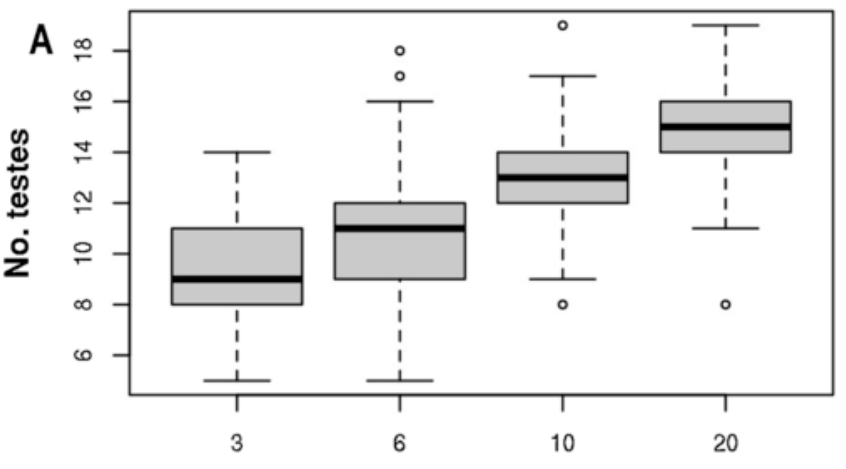

Amount of food

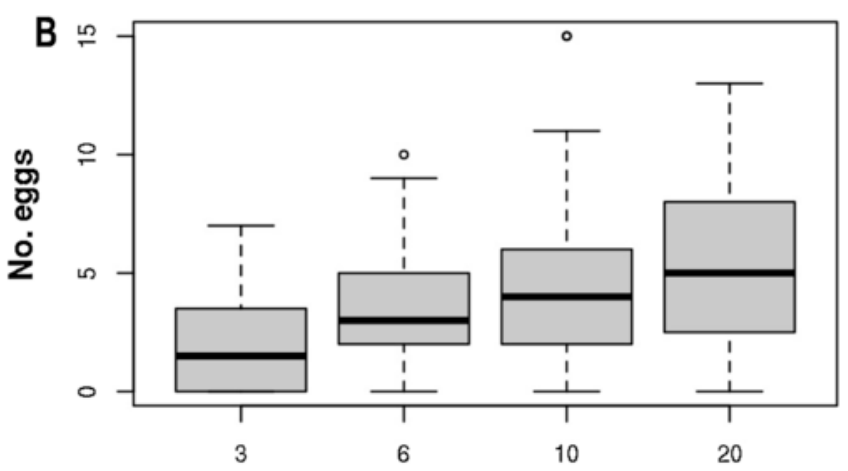

Amount of food

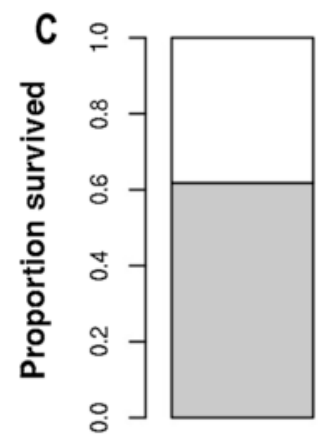

3

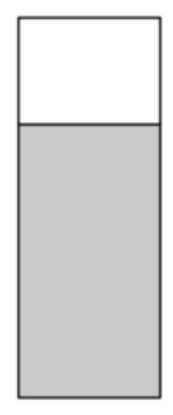

6

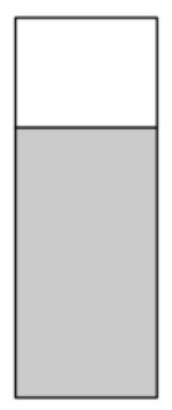

10

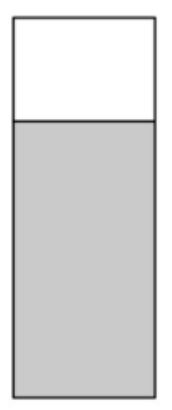

20

\section{Amount of food}

Fig. 4. Effects of food treatment on (A) the maximum number of testes produced by males, $(\mathrm{B})$ the number of eggs produced by females, and $(\mathrm{C})$ the survival rate.

\section{Discussion}

In the present study we investigated the fecundity/survival trade-off at different food levels in male and female H. oligactis undergoing sexual reproduction. We found that polyps initiated gonadogenesis irrespective of food availability, but the number of gonads (testes or detached eggs) increased with increasing food availability. Following sexual reproduction there was high mortality ( $>30 \%$ of the initial cohort died during the course of the experiment), but the survival rate was not related to the amount of food available.

Sexual reproduction in $H$. oligactis is generally thought to be an escape strategy that enables hydra to produce resting eggs before the onset of the winter (Reisa, 1973; Kaliszewicz and Lipińska, 2012). According to this hypothesis, a sudden drop in temperature signals the beginning of a period with high mortality due to low temperatures and/or starvation, and animals are induced to quickly develop reproductive organs and produce eggs that are able to survive these adverse conditions. Our observations suggest that this hypothesis cannot fully explain the occurrence of sexual reproduction in the studied population. First, lowering the temperature inhibited asexual reproduction only temporarily and the sexual phase started after a period of asexual reproduction in the cold. This suggests that temperature is a cue rather than a physiological constraint that shapes the type of reproduction (Reisa, 1973); the change in temperature could serve to indicate the time when sexual reproduction is ecologically most favored. This could be in the autumn, as supported by field observations of sexual reproduction in this species in Switzerland (Ribi et al., 1985) and Michigan, USA (Miller, 1936), but is probably later in our Hungarian population (based on observations in the present study). Secondly, similar to the study of Kaliszewicz and Lipińska (2012), the amount of food in our experiment had no influence on the probability of initiating sexual reproduction. Together, these observations suggest that the initiation of sexual reproduction in $H$. oligactis is not related to the amount of food, although other aspects of food, such as its predictability, are known to influence hydra life history traits (Schaible et al., 2011; Rosa et al., 2016) and might be involved in this case as well.

The delayed initiation of sexual reproduction in animals exposed to cold and the high post-reproductive survival rate of $H$. oligactis polyps from our population is surprising. In most published accounts of sexual reproduction in this species (e.g., Brien, 1953; Yoshida et al., 2006; Kaliszewicz and Lipińska, 2012; Kaliszewicz, 2015), exposure to cold temperatures inhibits budding and initiates gametogenesis within 2-3 weeks, after which polyps face an exponential increase in mortality rate (Brien, 1953; Yoshida et al., 2006). The experimental animals in the present study experienced progressive degeneration and a decrease in body size after sexual reproduction, very similar to that reported by Yoshida et al. (2006); however, a substantial proportion survived, regenerated and resumed asexual reproduction. Although these differences might be at least partly caused by differences in the experimental setup (e.g., both temperature and photoperiod differed between the studies), intraspecific differences in post-reproductive survival are likely to exist, as Tomczyk et al. (2015) recently also reported a strain of $H$. oligactis that does not experience increased post-reproductive mortality. The physiological mechanisms responsible for this variation are unclear. Post-reproductive degeneration in H. oligactis is generally thought to be the consequence of stem cell depletion ("gametic crisis"; Brien, 1953; Reisa, 1973; Tardent, 1974; Yoshida et al., 2006; Bosch, 2008). Sexual reproduction and germ cell development in hydra depend on stem cell activity (Littlefield, 1985, 1991), hence, increased commitment of stem cells to germ cell production is likely to reduce the differentiation of multipotent stem cells into somatic cells necessary for survival. The study of Yoshida et al. (2006) illustrates this pattern well, since they found a marked increase in germ cell numbers and a drastic decrease in epithelial cells, interstitial stem cells and somatic derivatives (nematocytes, nerve cells, and gland cells) during sexual development. Therefore, an obvious explanation for the high post-reproductive survival rate of the hydra population in the present study could be that in animals surviving sexual reproduction fewer stem cells are converted into germ cells and a sufficiently large subpopulation of stem cells remains to regenerate the whole animal. This is an assumption that should be tested in the future.

From an ultimate (evolutionary) perspective, differences between populations might be related to environmental conditions (e.g., the 
risk of freezing) in the natural habitats of these populations/strains. For example, a quick and predictable onset of winter would select for a rapid induction of gametogenesis upon cold exposure. Other factors are likely to be involved as well, because we found that within a single population several different life history strategies can coexist. Even within this admittedly very limited sample of 10 clones we found lineages that initiated sexual reproduction with high probability while others continued budding during the whole period (results not shown). Furthermore, some lineages survived with high probability while in others the survival rate was less than $25 \%$. These differences could represent alternative life history tactics within the population (e.g., local adaptations to differences in microhabitat) or a consequence of temporally varying selection on life history traits (e.g., Mojica et al., 2012). They could also be the consequence of a high rate of influx of genetically distinct individuals into the lake from which our founder polyps were derived. This latter hypothesis is highly plausible, given that the lake is very close to the Tisza river, is directly connected to it and periodically flooded by the river (the Tisza is a major river of the region with a large drainage area and is a known habitat of $\mathrm{H}$. oligactis; Gelei, 1930). Interestingly, we also found that polyps belonging to a single clone and kept under identical conditions exhibited variation in life history traits (e.g., some polyps did not initiate sexual reproduction, even though most individuals belonging to the same clones did), which could be a form of bet-hedging (diversifying reproductive strategies to increase the chance that some of them will be successful in a highly unpredictable environment; e.g., Olofsson et al., 2009).

Experimental manipulation of food availability significantly affected fecundity, but had no effect on the survival rate. Hence, at least over the range of food levels used in the present experiment, sexually reproducing $H$. oligactis polyps preserved survival at the expense of reproduction. This pattern is not consistent with a "dietary restriction" or "terminal investment" effect, but neither with a model in which survival and reproduction are equally dependent on food availability. On the other hand, a reduction in the rate of reproduction and maintenance of somatic functions in the face of challenging conditions has been observed, e.g., in reef corals (Leuzinger et al., 2011), and is generally predicted to occur in long-lived, iteroparous animals that are expected to favor survival over current reproduction (e.g., Gaillard et al., 2000; Therrien et al., 2008; Kitaysky et al., 2010; Monteith et al., 2013). This is somewhat surprising since $H$. oligactis is a species with a relatively fast life history (it is characterized by a high rate of asexual reproduction and low tolerance against oxidative stress; Tökölyi et al., 2016). Since most of the animals from the investigated population did not die after sexual reproduction, investing more resources into survival might enable them to survive and start asexual reproduction again following a sexual cycle (i.e., they might be iteroparous). Based on this hypothesis, the relationship between food availability and survival rate might be quite different in populations/strains that do not survive after sexual reproduction (i.e., semelparous populations). This is a prediction that should also be tested in the future.

Our results do not provide unequivocal support for the hypothesis that post-reproductive degeneration is a consequence of reduced allocation of resources to somatic maintenance in sexually reproducing hydra. First, the survival rate was not influenced by food availability (although, as discussed above, this could be a consequence of strategic investment of resources into survival at the expense of reproduction). Second, we found no relationship between fecundity and survival rate in individual polyps, which is a key prediction of the hypothesis that these two are traded off against each other. We must emphasize, however, that the variation in reproductive investment within our sample might not be high enough to detect such a relationship, since the indi- viduals used in the present analysis were derived from 10 clones and variation within clones might be too small. Further studies should (i) investigate the relationship between fecundity and survival rate on a much wider sample of hydra populations kept under identical conditions and (ii) attempt to manipulate reproductive investment independently from food (e.g., by manipulating mate availability or population density as ultimate factors, or potential physiological regulators as proximate factors) to unequivocally answer this question.

\section{Acknowledgements}

We are grateful to Csongor Freytag for his help in photographing hydra. Three anonymous reviewers provided comments that substantially improved the manuscript. We thank Renate Schilling for improving the English in this paper. This study was supported by the National Talent Program (NTP-EFÖ-P-15), by the Human Capacities Grant Management Office and the Hungarian Ministry of Human Capacities. J.T. was supported by the János Bolyai Research Scholarship of the Hungarian Academy of Sciences.

\section{References}

Bates, D., Maechler, M., Bolker, B., Walker, S., 2015. Fitting linear mixed-effects models using lme4. J. Stat. Softw. 67, 1-48.

Boehm, A.-M., Rosenstiel, P., Bosch, T.C.G., 2013. Stem cells and aging from a quasi-immortal point of view. BioEssays 35, 994-1003.

Boggs, C.L., 2009. Understanding insect life histories and senescence through a resource allocation lens. Funct. Ecol. 23, 27-37.

Bosch, T.C.G., 2008. Stem cells in immortal hydra. In: Bosch, T.C.G. (Ed.), Stem Cells: From Hydra to Man. Springer, Dordrecht, pp. 37-57.

Bridge, D., Theofiles, A.G., Holler, R.L., Marcinkevicius, E., Steele, R.E., Martínez, D.E., 2010. FoxO and stress responses in the cnidarian Hydra vulgaris. PLoS ONE 5, e11686.

Brien, P., 1953. La pérennité somatique. Biol. Rev. 28, 308-349.

Brock, M.A., Strehler, B.L., 1963. Studies on the comparative physiology of aging. IV. Age and mortality of some marine Cnidaria in the laboratory. J. Gerontol. $18,23-28$.

Clutton-Brock, T.H., 1984. Reproductive effort and terminal investment in iteroparous animals. Am. Nat. 123, 212-229.

Fischer, B., Taborsky, B., Dieckmann, U., 2009. Unexpected patterns of plastic energy allocation in stochastic environments. Am. Nat. 173, E108-E120.

Gaillard, J.-M., Festa-Bianchet, M., Yoccoz, N.G., Loison, A., Toïgo, C., 2000. Temporal variation in fitness components and population dynamics of large herbivores. Annu. Rev. Ecol. Syst. 31, 367-393.

Gelei, J., 1930. Tömlősök (Coelenterata). In: Brehm, A. (Ed.), Az állatok világa 18. (Alsórendú állatok II. kötet). Gutenberg Kiadó, Budapest, pp. 174-263. [in Hungarian].

Holstein, T.W., Hobmayer, E., Technau, U., 2003. Cnidarians: an evolutionarily conserved model system for regeneration?. Dev. Dyn. 226, 257-267.

Kaliszewicz, A., 2015. Intensity-dependent response to temperature in hydra clones. Zool. Sci. 32, 72-76.

Kaliszewicz, A., Lipińska, A., 2012. Maturation costs affect maturation timing: sexual reproduction in a heterogonic hydra. Hydrobiologia 679, 19-25.

Kirk, K.L., 2001. Dietary restriction and aging — comparative tests of evolutionary hypotheses. J. Gerontol. A Biol. Sci. Med. Sci. 56, B123-B129.

Kirkwood, T.B.L., Rose, M.R., 1991. Evolution of senescence: late survival sacrificed for reproduction. Phil. Trans. R. Soc. Lond. B 332, 15-24.

Kitaysky, A.S., Piatt, J.F., Hatch, S.A., Kitaiskaia, E.V., Benowitz-Fredericks, Z.M., Shultz, M.T., Wingfield, J.C., 2010. Food availability and population processes: severity of nutritional stress during reproduction predicts survival of long-lived seabirds. Funct. Ecol. 24, 625-637.

Leuzinger, S., Willis, B.L., Anthony, K.R.N., 2011. Energy allocation in a reef coral under varying resource availability. Mar. Biol. 159, 177-186.

Littlefield, C.L., 1985. Germ cells in Hydra oligactis males. Dev. Biol. 112, 185-193.

Littlefield, C.L., 1991. Cell lineages in Hydra: isolation and characterization of an interstitial stem cell restricted to egg production in Hydra oligactis. Dev. Biol. 143, 378-388.

Littlefield, C.L., Finkemeier, C., Bode, H.R., 1991. Spermatogenesis in Hydra oligactis. II. How temperature controls the reciprocity of sexual and asexual reproduction. Dev. Biol. 146, 292-300. 
Martıńez, D.E., 1998. Mortality patterns suggest lack of senescence in hydra. Exp. Gerontol. 33, 217-225.

Masoro, E.J., Austad, S.N., 1996. The evolution of the antiaging action of dietary restriction: a hypothesis. J. Gerontol. A Biol. Sci. Med. Sci. 51A, B387-B391.

McNamara, J.M., Houston, A.I., Barta, Z., Scheuerlein, A., Fromhage, L., 2009. Deterioration, death and the evolution of reproductive restraint in late life. Proc. Biol. Sci. 276, 4061-4066.

Miller, D.E., 1936. A limnological study of Pelmatohydra with special reference to their quantitative seasonal distribution. Trans. Am. Microsc. Soc. 55, 123-193.

Mojica, J.P., Lee, Y.W., Willis, J.H., Kelly, J.K., 2012. Spatially and temporally varying selection on intrapopulation quantitative trait loci for a life history trade-off in Mimulus guttatus. Mol. Ecol. 21, 3718-3728.

Monteith, K.L., Stephenson, T.R., Bleich, V.C., Conner, M.M., Pierce, B.M., Bowyer, R.T., 2013. Risk-sensitive allocation in seasonal dynamics of fat and protein reserves in a long-lived mammal. J. Anim. Ecol. 82, 377-388.

Nakagawa, S., Lagisz, M., Hector, K.L., Spencer, H.G., 2012. Comparative and meta-analytic insights into life extension via dietary restriction. Aging Cell 11, 401-409.

Olofsson, H., Ripa, J., Jonzén, N., 2009. Bet-hedging as an evolutionary game: the trade-off between egg size and number. Proc. Biol. Sci. 276, 2963-2969.

Partridge, L., Piper, M.D.W., Mair, W., 2005. Dietary restriction in Drosophila. Mech. Ageing Dev. 126, 938-950.

R Core Team, 2014. R: A Language and Environment for Statistical Computing. R Foundation for Statistical Computing, Vienna, Austria. https://R-project.org/.

Reisa, J., 1973. Ecology of Hydra. In: Burnett, A. (Ed.), Biology of Hydra. Academic Press, New York and London, pp. 59-105.

Ribi, G., Tardent, R., Tardent, P., Scascighini, C., 1985. Dynamics of hydra populations in Lake Zürich, Switzerland, and Lake Maggiore, Italy. Swiss J. Hydrol. 47, 45-56.

Rosa, M.E., Bradács, F., Tökölyi, J., 2016. Response of green hydra (Hydra viridissima) to variability and directional changes in food availability. Biologia (Bratisl.) 70, 1366-1375.
Schaible, R., Ringelhan, F., Kramer, B.H., Miethe, T., 2011. Environmental challenges improve resource utilization for asexual reproduction and maintenance in hydra. Exp. Gerontol. 46, 794-802.

Schaible, R., Sussman, M., Kramer, B.H., 2014. Aging and potential for self-renewal: hydra living in the age of aging - a mini-review. Gerontology 60, 548-556.

Schaible, R., Scheuerlein, A., Dańko, M.J., Gampe, J., Martínez, D.E., Vaupel, J.W., 2015. Constant mortality and fertility over age in hydra. Proc. Natl. Acad. Sci. U.S.A. 112, 15701-15706.

Schuchert, P., 2010. The European athecate hydroids and their medusae (Hydrozoa, Cnidaria): Capitata Part 2. Rev. Suisse Zool. 117, 337-555.

Schwentner, M., Bosch, T.C.G., 2015. Revisiting the age, evolutionary history and species level diversity of the genus Hydra (Cnidaria: Hydrozoa). Mol. Phylogenet. Evol. 91, 41-55.

Stelzer, C.-P., 2001. Resource limitation and reproductive effort in a planktonic rotifer. Ecology 82, 2521-2533.

Tardent, P., 1974. Gametogenesis in the genus Hydra. Am. Zool. 14, 447-456.

Therrien, J.-F., Côté, S.D., Festa-Bianchet, M., Ouellet, J.-P., 2008. Maternal care in white-tailed deer: trade-off between maintenance and reproduction under food restriction. Anim. Behav. 75, 235-243.

Tökölyi, J., Bradács, F., Hóka, N., Kozma, N., Miklós, M., Mucza, O., Lénárt, K., Ősz Z., Sebestyén, F., Barta, Z., 2016. Effects of food availability on asexual reproduction and stress tolerance along the fast-slow life history continuum in freshwater hydra (Cnidaria: Hydrozoa). Hydrobiologia 766, 121-133.

Tomczyk, S., Fischer, K., Austad, S., Galliot, B., 2015. Hydra, a powerful model for aging studies. Invertebr. Reprod. Dev. 59, 11-16.

Velando, A., Drummond, H., Torres, R., 2006. Senescent birds redouble reproductive effort when ill: confirmation of the terminal investment hypothesis. Proc. R. Soc. Lond. B 273, 1443-1448.

Walker, G., Houthoofd, K., Vanfleteren, J.R., Gems, D., 2005. Dietary restriction in C. elegans: from rate-of-living effects to nutrient sensing pathways. Mech. Ageing Dev. 126, 929-937.

Yoshida, K., Fujisawa, T., Hwang, J.S., Ikeo, K., Gojobori, T., 2006. Degeneration after sexual differentiation in hydra and its relevance to the evolution of aging. Gene 385, 64-70. 